\title{
Abnormal expression and mutation of the RBPJ gene may be involved in CD59- clonal proliferation in paroxysmal nocturnal hemoglobinuria
}

\author{
LIYAN LI* ${ }^{*}$, HUI LIU* ${ }^{*}$ HONGLEI WANG, ZHAOYUN LIU, YINGYING CHEN, \\ LIJUAN LI, JIA SONG, GUOJIN WANG and RONG FU \\ Department of Hematology, Tianjin Medical University General Hospital, Tianjin 300052, P.R. China
}

Received September 11, 2018; Accepted February 28, 2019

DOI: $10.3892 /$ etm.2019.7475

\begin{abstract}
Paroxysmal nocturnal hemoglobinuria (PNH) is an acquired clonal proliferative disease of hematopoietic stem cells. Various gene mutations, including the phosphatidylinositol glycan anchor biosynthesis class A (PIG-A) gene, may contribute to the proliferation of PNH clones. In order to explore the mechanism of PNH clone proliferation, a study was performed on 13 patients with PNH who underwent whole exome sequencing. The frequency of mutations in these patients was explored, and an additional 30 patients with PNH were selected for analysis of cluster of differentiation 59-negative (CD59-) cells. The mRNA expression of 13 genes, which were selected based on their high frequency in patients with PNH and the fact that they met four screening conditions, was determined in these CD59- cells. Cell proliferation, apoptosis and cell cycle were evaluated upon knocking down the recombinant signal binding protein of immunoglobulin $\mathrm{\kappa J}$ region (RBPJ) gene in 5 patients in vitro. The detection rate of PIG-A gene mutation was $61.54 \%$ (8/13), and additional mutations in somatic genes were detected, including RBPJ, zinc finger protein 717, polycomb repressive complex 2 subunit and tet methylcytosine dioxygenase. Upon screening according to the mutation frequency and expression level, the present study focused on the RBPJ gene. The expression level of RBPJ in CD59- cells was apparently higher than that in $\mathrm{CD} 59^{+}$cells and normal controls which was significantly correlated with clinical data. Furthermore, the expression of RBPJ in PNH primary cells could be effectively inhibited by
\end{abstract}

Correspondence to: Dr Rong Fu, Department of Hematology, Tianjin Medical University General Hospital, Tianjin 300052, P.R. China

E-mail: florai@sina.com

*Contributed equally

Key words: paroxysmal nocturnal hemoglobinuria, second gene mutation, recombinant signal binding protein of immunoglobulin $\mathrm{\kappa J}$ region, small interfering RNA small interfering RNA-RBPJ. Once the expression of RBPJ decreased remarkably, the apoptotic rate increased gradually, while cell proliferation activity decreased with transfection time and cells were blocked in G0/G1 phase. In conclusion, mutations and abnormal expression of the RBPJ gene may participate in the abnormal proliferation of $\mathrm{PNH}$ clones.

\section{Introduction}

Paroxysmal nocturnal hemoglobinuria (PNH) is a non-malignant clonogenic disease originating from hematopoietic stem cells that is caused by somatic gene mutations $(1,2)$. The pathological defect of PNH is the abnormal synthesis of glycosyl phosphatidyl inositol (GPI) caused by phosphatidylinositol glycan anchor biosynthesis class A (PIG-A) gene mutations on the $\mathrm{X}$ chromosome, which leads to the reduction or deletion of GPI-anchored proteins that are connected to the membrane of blood cells [including cluster of differentiation (CD)55, CD59 and CD56] and eventually causes activation of the complement system, leading to symptoms of intravascular hemolysis $(3,4)$. The main clinical manifestations of $\mathrm{PNH}$ are chronic intravascular hemolysis, bone marrow failure, high-risk complications of thrombosis, renal failure and pulmonary hypertension (5-7).

Our previous study (8) sequenced the whole genome exon of CD59- cells derived from 13 patients with PNH and identified additional mutant genes such as cut-like homeobox 1 (CUX1), mixed-lineage leukemia 2 (MLL2), SUZ12, RB transcriptional corepressor 1 (RB1), mucin 4 (MUC4) and tet methylcytosine dioxygenase 2 (TET2), although not PIG-A. The functions of these genes are associated with the regulation of cell proliferation, differentiation, resistance to apoptosis, invasion and progression of tumor cells (9-11). This suggests that, although PNH is a benign clonal proliferative disease, its clonal composition has similar biological characteristics to leukemia and other tumors. The proliferation advantage of PNH clones may require the participation of gene defects other than PIG-A gene mutations, suggesting that there may be additional gene mutations in patients with PNH (12-15). Therefore, the present study performed 500-depth whole exome sequencing (WES) for 13 patients with PNH. The mutation rate of the PIG-A gene was $30.77 \%$ (4/13) in 100-depth sequencing, whereas recombinant signal binding protein of immunoglobulin $\mathrm{\kappa J}$ region 
(RBPJ) gene mutation was detected in 6 patients $(46.15 \%)$. However, in 500-depth sequencing, the mutation rate of PIG-A was higher than in 100-depth sequencing $(61.54 \%, 8 / 13)$, which was consistent with literature reports, and 10 patients (76.92\%) exhibited RBPJ gene mutations. In-depth analysis and screening of the WES results were conducted. The present study screened out the RBPJ gene with high expression and first mutation level. It has been reported that RBPJ serves a key role in promoting the occurrence and development of various malignant tumors. Thus, inhibiting the expression of RBPJ may impair or promote the growth of potential tumors $(16,17)$. However, to the best of our knowledge, there have been no previous studies on the application of RNA interference to knock down the expression of RBPJ in patients with PNH.

The current study evaluated the regulation of PNH primary cells by RBPJ depletion. Small interfering RNA (siRNA) was used to efficiently knock down the RBPJ gene in PNH clones of 5 patients in vitro, and cell proliferation, apoptosis rate and cell cycle changes were observed to validate its functions.

\section{Materials and methods}

Patients. The clinical characteristics of the initial 13 patients with PNH, who were recruited from January-December 2015 and underwent WES, are presented in Table I. The results of WES were analyzed, 13 target genes were screened, and an additional 30 patients with $\mathrm{PNH}$ were recruited from January 2016-December 2017 for PCR experiments. The clinical characteristics of the additional 30 patients are presented in Table II. An Automatic hematology Instrument (LH 750; Beckman Coulter, Inc.) and an Automatic biochemical Instrument (C8000; Abbott Pharmaceutical Co., Ltd.) were utilized to detect blood cell counts [white blood cell (WBC), hemoglobinuria ( $\mathrm{Hb})$, platelet and reticulocyte (Ret)] and hemolysis indices [lactate dehydrogenase (LDH), total bilirubin and direct bilirubin), respectively. All patients were diagnosed with PNH and were admitted to the Department of Hematology of Tianjin Medical University General Hospital (Tianjin, China). The diagnostic criteria refer to the international PNH Research Group criteria (18). The control group consisted of 30 healthy donors, which were recruited from the Physical Examination Center of General Hospital of Tianjin Medical University from January 2017 to December 2017. Healthy controls (19 males and 11 females) were matched by sex and age, with an average age of 42 years (age range, 19-68 years). The Ethics Committee of Tianjin Medical University approved the present study (approval no. IRB2018-YX-042), and all the enrolled cases provided written informed consent.

Exome sequencing, reference genome and target area information. A total of 13 blood samples and paired normal fingernail samples were subjected to WES. In order to enrich the coding regions as much as possible, Sure Select Human All Exon 50M (Agilent Technologies, Inc.) was used for blood samples and Sure Select Human All Exon V4 (Agilent Technologies, Inc.) for nail samples. High-throughput sequencing of blood and nail samples was performed using an Illumina HiSeq2500 instrument (Illumina, Inc.). The average sequencing depths for blood and nail samples were 100x (range, 47-107x) and 102x (range, 96-106x), respectively. The sequencing depth of the above genes is presented in Fig. 1A. Broadband Wireless Access (BWA) was used to perform the alignment with the default parameters and human genome 19/the Genome Reference Consortium Human Genome Build 37 (hg19/GRCh37; ftp://ftp. ncbi.nlm.nih.gov/genomes/H_sapiens) served as the reference genome. The relevant details were as follows: Genome version number, hg19/GRCh37; genome size, 3095677412 bp; capture chip, SeqCap EZ Human Exome Library NimbleGen v2.0; exon sequencing genes $>20,000$ genes; and capture target area size, 44.1 Mb. The associated $1000 \mathrm{G}$ database was utilized to annotate and report mutations in MAF (https://www.ncbi.nlm. nih.gov/variation/tools/1000genomes/).

Flow cytometry and cell sorting. To obtain CD59- and CD59+ neutrophils for RNA extraction, cell sorting was performed in peripheral blood of patients with $\mathrm{PNH}$ and healthy controls on the same day of blood extraction. Red blood cells were lyzed with $10 \mathrm{ml}$ erythrocytolysin solution (BD Biosciences) and then centrifuged at $150 \mathrm{x} g$ for $5 \mathrm{~min}$ at room temperature. Cells were then washed twice with PBS and resuspended in $300 \mu \mathrm{l}$ PBS. Immunomagnetic cell selection was performed using phycoerythrin (PE) conjugated-anti-CD59 Phycoerythrin (1:5; cat. no. 555764; BD Biosciences), followed by anti-PE microbeads (Miltenyi Biotec $\mathrm{GmbH}$ ). Samples were separated using MS Columns (Miltenyi Biotec $\mathrm{GmbH}$ ), and purity was verified in each fraction by flow cytometry using an Aria II instrument (BD Biosciences). All results were analyzed using CellQuest $^{\mathrm{TM}}$ Pro Software 4.0.2 (BD Biosciences).

To ensure the viability of PNH cells in culture and the transfection efficiency, CD59- of neutrophils from 15 patients with PNH were sorted by flow cytometry. Upon cell sorting, the number of cells reached $2 \times 10^{7}$, and the purity of the cells was $96-97 \%$.

Reverse transcription-quantitative polymerase chain reaction $(R T-q P C R)$. Total RNA was extracted from CD59- and CD59+ cells of patients with $\mathrm{PNH}$ and normal controls, and from mononuclear cells, respectively, with RNeasy kit (Takara Bio, Inc.). A Reverse transcription kit (Takara Bio, Inc.) was used to synthesize cDNA from $1 \mu \mathrm{g}$ total RNA and was purified using the QIAquick PCR Purification Kit (Qiagen, Inc.). QuantiTect SYBR Green PCR Kit (Tli RNaseH Plus; Takara Bio, Inc.) and Light Cycler 1.5 Real-Time PCR System (Roche Diagnostics, Indianapolis, IN, USA) were used to perform RT-qPCR in duplicates. Specific primers designed to amplify cross-exons of the RBPJ gene (158 bp) are detailed in Table III. The thermocycling conditions were as follows: Initial denaturation at $95^{\circ} \mathrm{C}$ for 30 sex; followed by 45 cycles of denaturation at $94^{\circ} \mathrm{C}$ for $5 \mathrm{sec}$, annealing at $60^{\circ} \mathrm{C}$ for $30 \mathrm{sec}$ and extension at $70^{\circ} \mathrm{C}$ for $30 \mathrm{sec}$. A final extension was conducted at $72^{\circ} \mathrm{C}$ for $10 \mathrm{~min}$. Bio-Rad CFX Manager software 3.1 (Bio-Rad Laboratories, Inc.) was used to analyze the melting and amplification curves (quantitative curve), and the quantitative cycle $(\mathrm{Cq})$ values of each group was determined. The relative quantitative multiplier of each group (relative fold) was expressed by $2^{-\Delta \Delta C q}$ value (19) and used for statistical analysis.

Cell culture. CD59- neutrophils were inoculated into RPMI-1640 culture medium (Beijing Solarbio Science \& 
Technology Co., Ltd.) containing 10\% (v/v) fetal bovine serum (Beijing Solarbio Science \& Technology Co., Ltd.), penicillin $(100 \mathrm{U} / \mathrm{ml})$ and streptomycin $(100 \mathrm{U} / \mathrm{ml})$, and were suspended in a saturated humidity chamber containing $5 \%$ $\mathrm{CO}_{2}$ at $37^{\circ} \mathrm{C}$. The centrifugal fluid exchange method $(1.4 \mathrm{x} \mathrm{g}$ for $5 \mathrm{~min}$ at room temperature) was used to replace the medium every $48 \mathrm{~h}$ and maintain strict aseptic conditions. Logarithmic growth phase cells were used for the experiments if their rejection rate of trypan blue (Beijing Solarbio Science \& Technology Co., Ltd.) staining was $>95 \%$. The steps of trypan blue staining were as follows: Cell suspension $(0.1 \mathrm{ml})$ was added into $4 \mathrm{ml}$ trypan blue solution (final dilution, 1:40) and placed at room temperature for 3-5 min. A drop of cell suspension was taken and placed on a slide. The number of living and dead cells in 1,000 cells was subsequently counted. From these results, cell viability was calculated as follows: Cell viability $(\%)=$ number of unstained cells/total number of observed cells x100. Following $72 \mathrm{~h}$ of cell culture, cell count reached $10^{7}$ cells.

Preparation of RBPJ-siRNAs and transfection. Specific siRNAs of the RBPJ gene (siRNA-RBPJ1, siRNA-RBPJ2 and siRNA-RBPJ3) were purchased from Genomeditech Co., Ltd., whereas siRNA-scramble (scr) served as an internal reference (Genomeditech Co.,Ltd.). The siRNA sequences are provided in Table III. All transfections with siRNAs were performed using Lipofectamine $^{\mathrm{TM}} 3000$ (lipo3000; cat. no. 100022234; Thermo Fisher Scientific, Inc., Waltham, MA, USA). siRNA-RBPJ and lipo3000 were diluted with Opti-MEM (Invitrogen; Thermo Fisher Scientific, Inc.). In order to achieve a high transfection efficiency and gene blocking effect, siRNAs were fluorescently labeled (Dextran, Cy7 labeled, Genomeditech Co., Ltd.), and concentration gradient experiments were conducted on siRNAs (Genomeditech Co., Ltd.) and lipo3000 (cat. no. 100022234; Thermo Fisher Scientific, Inc.). The experimental steps of the concentration gradient were as follows: A 24-well plate (4-8x $10^{5}$ cells/well) was used to perform concentration gradient cell transfection experiments. The final concentration of siRNA and lipofectamine ${ }^{\mathrm{TM}} 3000$ was $30 \mathrm{nM}$ (siRNA, $0.75 \mu \mathrm{l}$; lipofectamine $\mathrm{TM}^{\mathrm{TM}} 3000,1 \mu \mathrm{l}$ ), $50 \mathrm{nM}$ (siRNA, $1.25 \mu \mathrm{l}$, lipofectamine $\left.\mathrm{TM}^{\mathrm{TM}} 3000,1 \mu \mathrm{l}\right)$ and $100 \mathrm{nM}$ (siRNA, $2.5 \mu \mathrm{l}$, lipofectamine $\left.^{\mathrm{TM}} 3000,1 \mu \mathrm{l}\right)$. The silencing efficiency of siRNA was detected by PCR $48 \mathrm{~h}$ following transfection. When cells were co-transfected with lipo3000 and RBPJ-specific siRNAs at $50 \mathrm{nM}$ (lipo3000, $1 \mu \mathrm{l} /$ well; siRNA1, $1.25 \mu \mathrm{l} /$ well; total volume of medium, $500 \mu \mathrm{l} /$ well), the siRNA was capable of efficiently blocking the expression of the target gene (siRNA silencing efficiency was detected using RT-qPCR $48 \mathrm{~h}$ following transfection). The transfection efficiency of siRNA to the cultured cells was revealed to be $75-80 \%$. After the above preliminary experiments, siRNA1 was used for subsequent experiments following validation of transfection. A 24-well plate was used for the cell transfection experiment. At $24 \mathrm{~h}$ prior to transfection, $2 \times 10^{5}$ cells were inoculated into $400 \mu \mathrm{l}$ Opti-MEM to ensure that the cell density reached $4-8 \times 10^{5}$ cells/well at the time of transfection. The diluent of lipo3000 and siRNA was gently mixed to guarantee a final concentration of $50 \mathrm{nM}$. The mixture was incubated at room temperature for $20 \mathrm{~min}$ and then added to the 24 -well plate at $100 \mu \mathrm{l} /$ well and mixed evenly. The plates were incubated for $18-48 \mathrm{~h}$ at $37^{\circ} \mathrm{C}$ and $5 \% \mathrm{CO}_{2}$. At
Table I. Initial clinical characteristics of $13 \mathrm{PNH}$ patients.

\begin{tabular}{|c|c|}
\hline Clinical feature & Measurement \\
\hline \multicolumn{2}{|l|}{ Clinical classification of PNH, n (\%) } \\
\hline Classical PNH & $7(53.85)$ \\
\hline PNH-AA & $6(46.15)$ \\
\hline Subclinical-PNH & 0 \\
\hline \multicolumn{2}{|l|}{ Sex, n $(\%)$} \\
\hline Male & $6(46.15)$ \\
\hline Female & $7(53.85)$ \\
\hline Age (years), median age (range) & $31(21-73)$ \\
\hline \multicolumn{2}{|l|}{ Blood examination } \\
\hline RET \% & $7.98 \pm 4.57$ \\
\hline $\mathrm{RBC}\left(* 10^{12} / 1\right)$ & $2.44 \pm 0.63$ \\
\hline $\mathrm{WBC}\left(* 10^{9} / 1\right)$ & $3.94 \pm 2.14$ \\
\hline $\operatorname{HGB}(\mathrm{g} / \mathrm{l})$ & $82.08 \pm 20.24$ \\
\hline $\operatorname{PLT}\left(* 10^{9} / 1\right)$ & $73.46 \pm 66.66$ \\
\hline D-Dimer (ug/ml) & $1,672.00 \pm 1,404.00$ \\
\hline $\mathrm{LDH}(\mathrm{U} / \mathrm{l})$ & $1,058.00 \pm 663.30$ \\
\hline TBIL $(\mu \mathrm{mol} / 1)$ & $24.48 \pm 14.69$ \\
\hline DBIL $(\mu \mathrm{mol} / \mathrm{l})$ & $7.18 \pm 3.59$ \\
\hline Granulocyte CD59. (\%) & $78.58 \pm 17.02$ \\
\hline Erythrocyte CD59.(\%) & $52.53 \pm 30.28$ \\
\hline Flaer/CD14- (\%) & $81.52 \pm 12.63$ \\
\hline Flaer/CD24- (\%) & $85.52 \pm 13.29$ \\
\hline
\end{tabular}

Data are presented as the mean \pm standard deviation unless otherwise stated. PNH, paroxysmal nocturnal hemoglobinuria; AA, Aplastic anemia; RET, reticulocyte ratio; RBC, red blood cell; WBC, white blood cell; HGB, hemoglobin; PLT, platelet; TBIL, total bilirubin; DBIL, direct bilirubin; LDH, lactate dehydrogenase; Flaer, fluorescent aerolysin; $\mathrm{CD}$, cluster of differentiation.

4-6 h post-transfection, the culture medium was replaced with fresh medium.

Cell Counting Kit-8 (CCK-8) assay for cell proliferation. A hemocytometer was used to count the number of cells in the prepared cell suspension, and then the cells were inoculated in 96-well plate (100 $\mu \mathrm{l} /$ well). Following $4 \mathrm{~h}$ of cell culture, CCK-8 reagent (Beijing Solarbio Science \& Technology Co., Ltd.) was added to the culture for a certain time (5-10 min at room temperature) and then the optical density (OD) value at $450 \mathrm{~nm}$ of was determined. A standard curve was created depicting the number of cells (x-axis) vs. the OD value (y-axis). Subsequently, the cell proliferation activity was determined by adding $10 \mu \mathrm{l}$ CCK-8 solution to the 96 -well plates containing $100 \mu \mathrm{l}\left(5 \times 10^{4}\right.$ cells $\left./ \mathrm{ml}\right)$ cell suspension per well. Blank control wells were prepared at the same time. Next, the cells were incubated for $4 \mathrm{~h}$ under $5 \% \mathrm{CO}_{2}$ and $37^{\circ} \mathrm{C}$ conditions. The OD value at $450 \mathrm{~nm}$ was determined using a Microplate Reader (cat. no. ELX800; BioTek Instruments, Inc.). The median value was obtained by repeating the experiment 3 times and using the following formula: Cell proliferation activity=OD value of the experimental wells-OD value of the control wells; 
Table II. Initial clinical characteristics of $30 \mathrm{PNH}$ patients.

\begin{tabular}{|c|c|c|c|}
\hline Characteristic & Patients & Controls & P-value \\
\hline Total, n & 30 & 30 & \\
\hline Gender, male/female & $19 / 11$ & $19 / 11$ & \\
\hline Age (years), median (range) & $38(18-75)$ & $42(19-68)$ & \\
\hline Clinical classification, n (\%) & & 0 & \\
\hline Classical PNH & $23(76.67)$ & & \\
\hline PNH-AA & $6(20.00)$ & & \\
\hline Subclinical-PNH & $1(3.33)$ & & \\
\hline History of thrombosis, n (\%) & $4(13.33)$ & 0 & \\
\hline \multicolumn{4}{|l|}{ Parameters at baseline } \\
\hline $\operatorname{HGB}(g / 1)$ & $78.71 \pm 20.94$ & $127.30 \pm 9.64$ & $<0.001$ \\
\hline $\operatorname{Ret}(\%)$ & $9.85 \pm 5.24$ & $1.34 \pm 0.33$ & 0.0017 \\
\hline WBC $\left(\times 10^{9} / 1\right)$ & $5.89 \pm 3.55$ & $6.11 \pm 1.51$ & 0.778 \\
\hline PLT (x109/1) & $100.40 \pm 65.77$ & $171.80 \pm 56.06$ & 0.003 \\
\hline TBIL $(\mu \mathrm{mol} / 1)$ & $31.79 \pm 15.07$ & $10.75 \pm 6.11$ & 0.016 \\
\hline $\mathrm{DBIL}(\mu \mathrm{mol} / \mathrm{l})$ & $8.34 \pm 3.54$ & $3.77 \pm 2.17$ & 0.045 \\
\hline $\mathrm{LDH}(\mathrm{U} / \mathrm{l})$ & $1,578.00 \pm 931.70$ & $191.50 \pm 228.70$ & $<0.001$ \\
\hline $\mathrm{Cr}(\mu \mathrm{mol} / 1)$ & $60.19 \pm 27.47$ & $53.35 \pm 21.67$ & 0.531 \\
\hline Granulocyte CD59- (\%) & $80.44 \pm 17.30$ & $1.84 \pm 1.05$ & $<0.001$ \\
\hline Erythrocyte CD59- (\%) & $52.38 \pm 30.25$ & $2.69 \pm 1.27$ & 0.006 \\
\hline Flaer-CD14- (\%) & $77.30 \pm 21.28$ & Undetected & \\
\hline Flaer/CD24- (\%) & $82.65 \pm 17.62$ & Undetected & \\
\hline
\end{tabular}

Data are presented as the mean \pm standard deviation unless otherwise stated. PNH, paroxysmal nocturnal hemoglobinuria; AA, Aplastic anemia; RET, reticulocyte ratio; WBC, white blood cell; HGB, hemoglobin; PLT, platelet; TBIL, total bilirubin; DBIL, direct bilirubin; LDH, lactate dehydrogenase; $\mathrm{Cr}$, creatinine; Flaer, fluorescent aerolysin; $\mathrm{CD}$, cluster of differentiation.

where the OD value of the experimental wells represents the absorption of cells and CCK-8 solution, while the OD value of the control wells represents the absorption of the well with medium and CCK-8 solution.

Cell cycle analysis. A cell suspension (1 $\mathrm{ml}$ containing $1 \times 10^{6}$ cells $/ \mathrm{ml}$ ) was obtained following transfection for $72 \mathrm{~h}$ by washing the cells 2 times with $1 \mathrm{ml}$ PBS pre-cooled at $4^{\circ} \mathrm{C}$, followed by centrifugation $(150 \mathrm{x}$ g for $5 \mathrm{~min}$ at room temperature) of the supernatant. PBS was added to the cell suspension and incubated with $70 \%$ ethanol at $4^{\circ} \mathrm{C}$ for pre-cooling. Following $4 \mathrm{~h}$ of incubation, all samples were separated by centrifugation (300 x g for $5 \mathrm{~min}$ at room temperature). Next, $500 \mu \mathrm{l}$ cell cycle dye (BD Pharmingen; Becton, Dickinson and Company, Franklin Lakes, NJ, USA) was added to the cell suspension and incubated for 15-20 min at room temperature. Flow cytometry analysis (CellQuest ${ }^{\mathrm{TM}}$ Pro Software 4.0.2; BD Biosciences) was then used to detect the relative content of DNA in the cells, and the results of DNA distribution of cell proportion in the phase of cell cycle were analyzed by Modifit (Verify Software House Inc, Topsham, ME, USA) with a propidium iodide kit (PI, $20 \mu \mathrm{g} / \mathrm{ml})$ containing RNase A (10 $\mu \mathrm{g} / \mathrm{ml}$; BD Biosciences). The percentage of DNA in each phase of cell cycle was analyzed to reflect the proliferation status of the cells and the content of DNA in non-diploid (aneuploid) cells. The DNA content of G2 and M-phase cells is typically double that exhibited by cells in the G0 and G1 phases of the cell cycle, while the DNA content of S-phase cells is intermediate between these values (20).

Apoptosis. Following 24, 48 or $72 \mathrm{~h}$ of culture, the cells were washed twice with cold PBS and resuspended in 1X Binding Buffer (BD Pharmingen; Becton, Dickinson and Company) at a concentration of $1 \times 10^{6}$ cells $/ \mathrm{ml}$. A total of $100 \mathrm{ml}$ solution $\left(1 \times 10^{5}\right.$ cells $)$ was transferred to a $5-\mathrm{ml}$ culture tube. Then, $5 \mu \mathrm{l}$ fluorescein isothiocyanate (FITC)-Annexin V (BD Pharmingen; Becton, Dickinson and Company) and $5 \mu \mathrm{l}$ propidium iodide (PI; BD Pharmingen; Becton, Dickinson and Company) were added to the cells, followed by gently mixing at room temperature and subsequent incubation for $15 \mathrm{~min}$ in the dark. Next, $400 \mathrm{ml}$ 1X Binding Buffer was added to each test tube. Flow cytometry analysis (CellQuest ${ }^{\mathrm{TM}}$ Pro Software 4.0.2 (BD Biosciences) was performed within $1 \mathrm{~h}$. Surviving cells were FITC-Annexin V and PI-negative; early apoptotic cells were FITC-Annexin V-positive and PI-negative; and late apoptotic or dead cells were FITC-Annexin V and PI-positive.

Statistical analysis. SPSS 23.0 statistical software (IBM Corp., Armonk, NY, USA) was used for statistical analysis of the data. Data are presented as the mean \pm standard deviation, and the standard deviation was used to estimate the sample distribution. Non-normally distributed data comparisons between groups were performed via one-way analysis, and Tukey's 

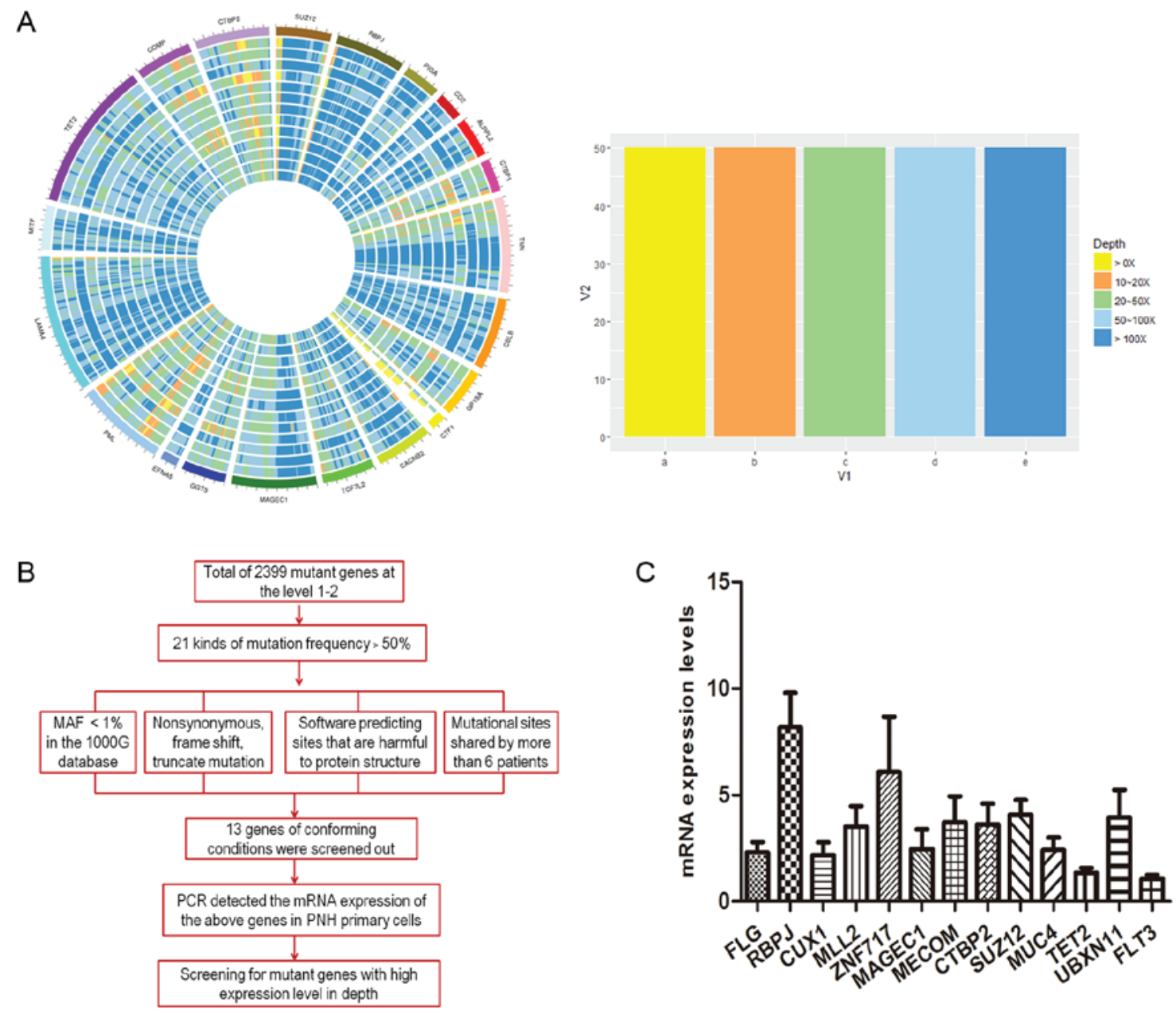

D
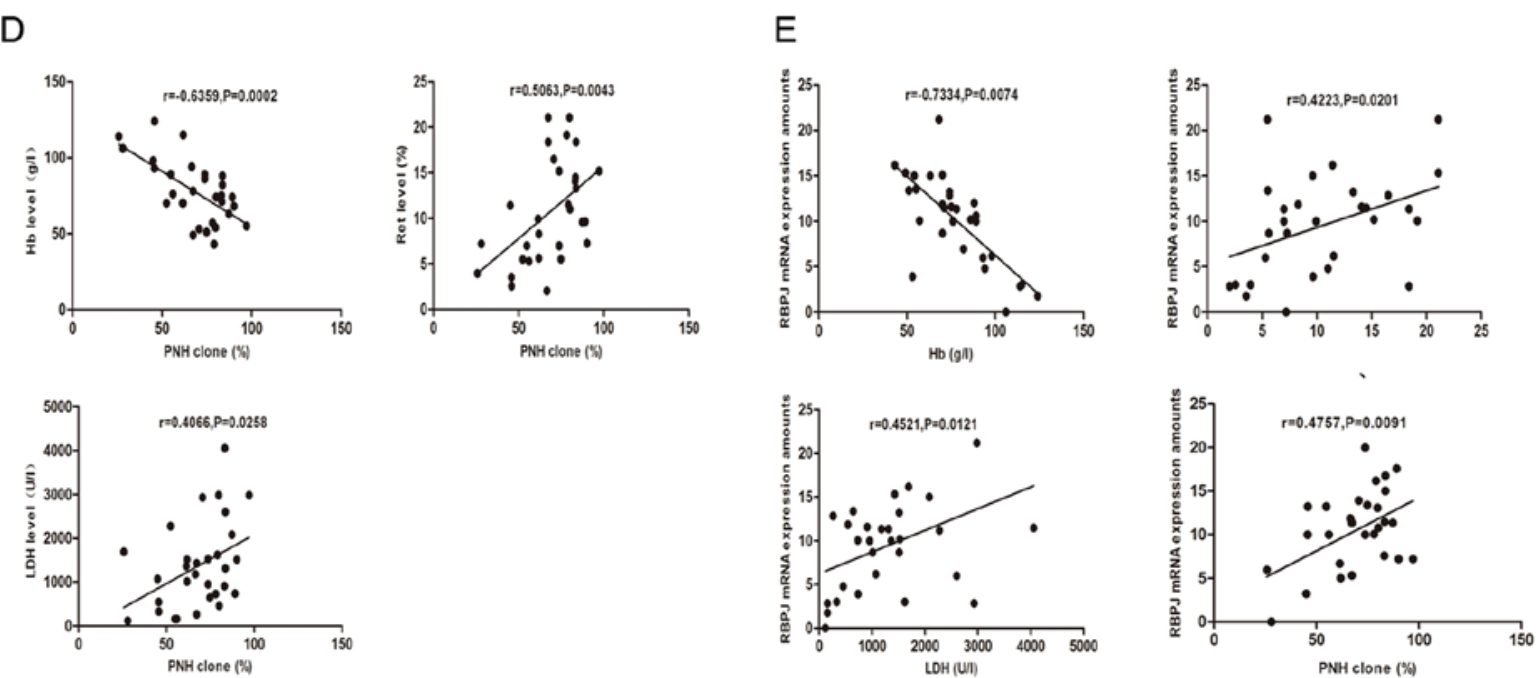

Figure 1. (A) The outermost color represents different genes, different colors represent different sequencing depth, each gene width represents the length of the corresponding gene coding region. (B) Flow chart of screening target genes. (C) Comparison of relative expression levels of 13 target mutant genes in $30 \mathrm{PNH}$ patients which were screened by whole exome sequencing. (D) Correlation analysis between PNH cloning proportion and hemolysis index. (E) Correlation analysis between RBPJ mRNA expression and clinical hemolysis index. The expression of RBPJ mRNA was negatively correlated with the proportion of the level of $\mathrm{Hb}$, and positively correlated with the proportion of RET, LDH levels and the proportion of PNH clones. PNH, paroxysmal nocturnal hemoglobinuria; RPBJ, recombinant signal binding protein of immunoglobulin $\mathrm{\kappa J}$ region; $\mathrm{Hb}$, hemoglobinuria; RET, reticulocyte; LDH, lactate dehydrogenase; FLG, filaggrin; CUX1, cut-like homeobox 1; MLL2, mixed-lineage leukemia 2; ZNF717, zinc finger protein 717; MAGEC1, MAGE family member C1; MECOM, MDS1 and EVI1 complex locus; CTBP2, C-terminal binding protein 2; MUC4, mucin 4; TET2, tet methylcytosine dioxygenase 2; UBXN11, UBX domain protein 11; FLT3, fms related tyrosine kinase 3; MAF, minor allele frequency; PCR, polymerase chain reaction.

honestly significant difference was used as the post hoc test for multiple comparisons. Spearman's correlation analysis was used to evaluate the association between qualitative variables.
Paired Student's t-test was used for pairwise comparison between groups. $\mathrm{P}<0.05$ was considered to indicate a statistically significant difference. 
Table III. Gene primer and siRNA sequences.

\begin{tabular}{ll}
\hline Name & \multicolumn{1}{c}{ Sequence (5'-3') } \\
\hline RBPJ & Forward, AGTCACTCCTGTGCCTGTGGTAG \\
siRNA-RBPJ1 & Reverse, CCATCTCCAACCTTCTCGGAATGC \\
& Forward, GCACUCCCAAGAUU GAUAA \\
siRNA-RBPJ2 & Reverse, UUAUCAA UCUUGGGAGUGC \\
& Forward, GCACUCCCAAGAUU GAUAA \\
siRNA-RBPJ3 & Reverse, UUAUCAAUCUUGGGAGUGC \\
& Forward, CUGACUCAGACAAGCGAAA \\
siRNA-scr & Reverse, UUUCGCUUGUCUGAGU CAG \\
& Forward, UUGAAGUUAU GUAUCCUCC UU \\
GAPDH & Reverse, CUGAAGCUGCUGGGAGUAAUU \\
& Forward, GGAGCGAGATCCCTCCAAAAT \\
& Reverse, GGCTGTTGTCATACTTCTCATGG \\
\hline
\end{tabular}

siRNA, small interfering RNA; RPBJ, recombinant signal binding protein of immunoglobulin $\kappa \mathrm{J}$ region; scr, scrambled control.

Table IV. High expression of RBPJ mRNA in comparison with controls.

\begin{tabular}{lccc}
\hline Gene & CD59-ell group & Control group & P-value \\
\hline FLG & $2.310 \pm 1.193$ & $2.682 \pm 1.346$ & 0.624 \\
RBPJ & $8.177 \pm 3.974$ & $3.683 \pm 2.661$ & 0.044 \\
CUX1 & $2.182 \pm 1.454$ & $0.780 \pm 0.842$ & 0.067 \\
MLL2 & $3.517 \pm 2.377$ & $2.637 \pm 1.367$ & 0.450 \\
ZNF717 & $6.083 \pm 6.377$ & $8.137 \pm 7.183$ & 0.629 \\
MAGEC1 & $2.470 \pm 2.276$ & $2.803 \pm 2.788$ & 0.825 \\
MECOM & $3.737 \pm 2.946$ & $2.303 \pm 1.634$ & 0.322 \\
CTBP2 & $3.620 \pm 2.384$ & $6.453 \pm 4.262$ & 0.186 \\
SUZ12 & $4.087 \pm 1.655$ & $6.453 \pm 4.117$ & 0.022 \\
MUC4 & $2.413 \pm 1.438$ & $6.120 \pm 4.023$ & 0.041 \\
TET2 & $1.353 \pm 0.552$ & $5.067 \pm 4.675$ & 0.002 \\
UBXN11 & $3.940 \pm 3.201$ & $4.607 \pm 2.907$ & 0.714 \\
FLT3 & $1.067 \pm 0.471$ & $2.353 \pm 1.990$ & 0.006 \\
\hline
\end{tabular}

Data are presented as the mean \pm standard deviation. The relative expression of the gene is expressed by $2^{-\Delta \Delta \mathrm{Cq}}$ value. RPBJ, recombinant signal binding protein of immunoglobulin $\kappa \mathrm{J}$ region; FLG, filaggrin; CUX1, cut-like homeobox 1; MLL2, mixed-lineage leukemia 2; ZNF717, zinc finger protein 717; MAGEC1, MAGE family member $\mathrm{C} 1$; MECOM, MDS1 and EVI1 complex locus; CTBP2, C-terminal binding protein 2; MUC4, mucin 4; TET2, tet methylcytosine dioxygenase 2; UBXN11, UBX domain protein 11; FLT3, fms related tyrosine kinase 3.

\section{Results}

Screening of mutations in the target gene RBPJ from WES sequencing results. A total of 13 patients with $\mathrm{PNH}$ patients were sequenced by 500-depth, of which $61.54 \%$ (8/13) exhibited PIG-A gene mutations. The mutation rate of the PIG-A gene was $61.54 \%(8 / 13)$ in 500-depth sequencing, which was significantly higher than that of 100 -depth sequencing $(30.77 \%$; 4/13) and consistent with the literature (21). The mutation types mainly included frame shift, splicing, stop-gain and non-synonymous mutations.

The 500-depth sequencing results revealed additional somatic gene mutations other than those affecting PIG-A. According to the risk of mutation, it can be divided into five grades, of which 1-2 was the first mutation explored in the present study (22). There were 2,399 mutant genes with a mutation grade of 1 or 2 , and 21 had mutation frequencies $>50 \%$ [namely, kinesin family member 24, MAGE family member C1 (MAGEC1), fms related tyrosine kinase 3 (FLT3), mediator of DNA damage checkpoint 1, leukocyte immunoglobulin like receptor B3 (LILRB3), CUX1, zinc finger protein 717 (ZNF717), double homeobox 4 like 4, UBX domain protein 11 (UBXN11), teashirt zinc finger homeobox 1, filaggrin (FLG), trichohyalin, SUZ12, RBPJ, C-terminal binding protein 2 (CTBP2), MDS1 and EVI1 complex locus (MECOM), MUC4, MLL2, POTE ankyrin domain family member H, TET2, and NBPF member 1]. For the mutant genes mentioned above, the following screening requirements were also set according to the mutation forms and the degree of damage of the mutation sites to the structure of the encoding protein: i) Their mutation rate in the genome of 1,000 individuals in the $1000 \mathrm{G}$ database is $<1 \%$; ii) they contain splicing mutations, frame shifts or non-synonymous mutations; iii) the software predicts the harmful mutation sites in the protein structure; and iv) there are mutual mutation sites in $>6$ patients. A total of 13 mutant genes met the above conditions, including FLG, RBPJ, CUX1, MLL2, ZNF717, MAGEC1, MECOM, CTBP2, SUZ12, MUC4, TET2, UBXN11 and FLT3. Furthermore, 30 patients with PNH were selected, from whom the mRNA of CD59- cells was extracted, and the mRNA expression of the above 13 genes was detected by RT-qPCR (Fig. 1B). The correlation between PNH clones and clinical markers, relative expression of mutant gene and hemolysis markers was also analyzed. Ultimately, the target genes that were highly expressed and have a significant 
A

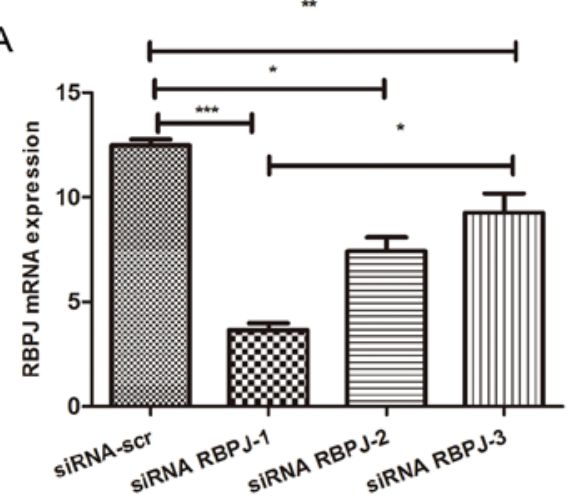

C

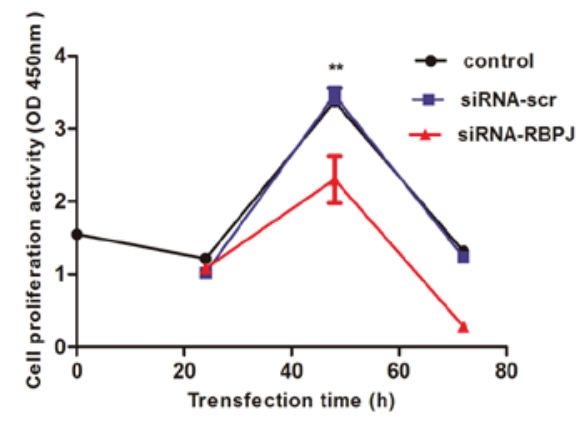

B

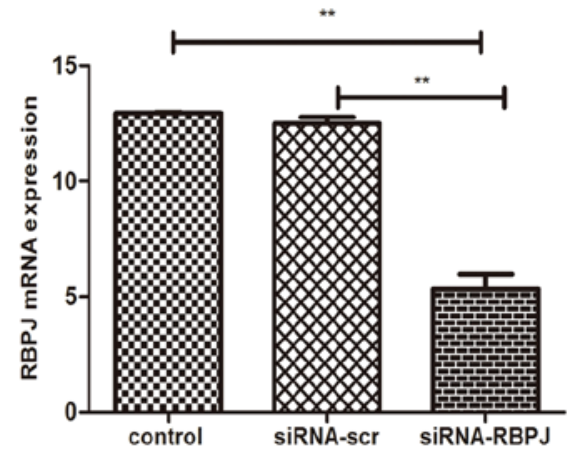

D

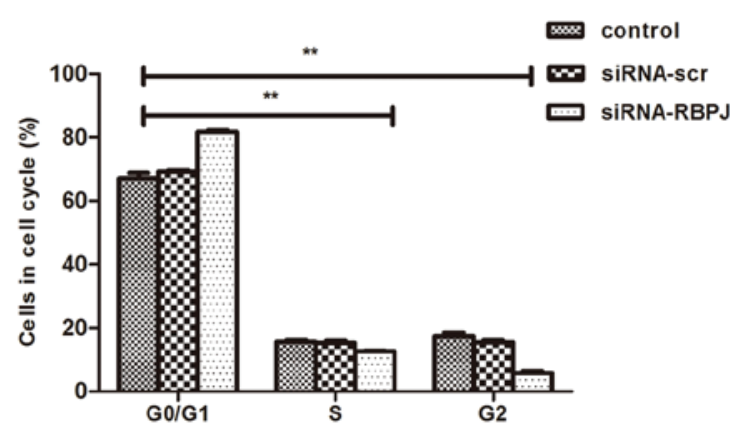

E
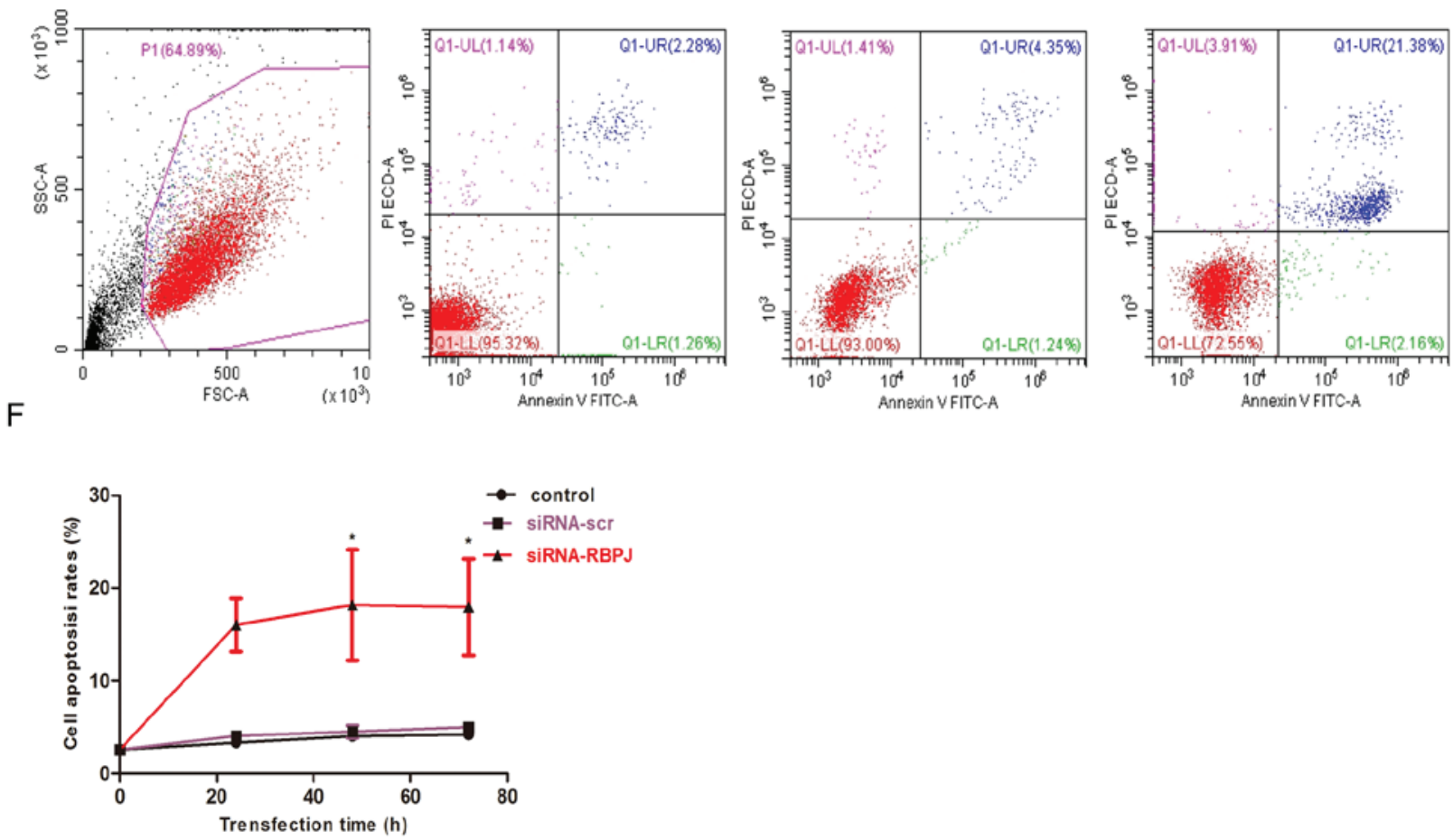

Figure 2. (A) Evaluation of the inhibitory effects of siRNAs on RBPJ expression. Quantified RT-qPCR data for RBPJ in PNH primary cells transfected with siRNA-RBPJ. (B) Inhibition of RBPJ by siRNA-RBPJ in stable PNH primary cells. PNH primary cells were infected with RBPJ-siRNA and scr-siRNA to generate stable clones which were then cultured in media for $72 \mathrm{~h}$ before they were harvested. RBPJ levels were significantly reduced in PNH primary cells. (C) Cell growth was examined via cell counting kit-8 assay. Significantly reduced cell growth via RBPJ inhibition was detected as early as $48 \mathrm{~h}$ after seeding, compared with controls and scr-siRNA. (D) PNH primary cells were analyzed in a cell cycle phase assay at $72 \mathrm{~h}$ after seeding. It was demonstrated that the percentage of S-phase proliferating cells and G2-phase post-replicating cells in RBPJ-depleted PNH primary cells was significantly reduced, whereas the percentage of G0/G1-phase cells in RBPJ-depleted cells was significantly increased, compared with control cells. (E and F) Cell apoptosis rate was examined by flow cytometry. Significantly increased cell apoptosis rate by RBPJ inhibition was detected as early as $24 \mathrm{~h}$ after seeding, compared with controls and scr-siRNA. " $\mathrm{P}<0.05,{ }^{* *} \mathrm{P}<0.01,{ }^{* * *} \mathrm{P}<0.001$. siRNA, small interfering RNA; scr, scrambled control; PNH, paroxysmal nocturnal hemoglobinuria; RPBJ, recombinant signal binding protein of immunoglobulin $\kappa \mathrm{J}$ region; OD, optical density.

correlation with PNH hemolysis index were selected to carry out the following experiments.
The results were as follows: The RBPJ gene was highly expressed, and the relative expression level of the RBPJ gene 
Table V. Comparison of proliferative activity and apoptosis rates of 24, 48 and $72 \mathrm{~h}$ before and after siRNA RBPJ transfection in paroxysmal nocturnal hemoglobinuria primary cells.

\begin{tabular}{lccccccc}
\hline & \multicolumn{3}{c}{ Proliferative activity $(\%)$} & & \multicolumn{3}{c}{ Apoptosis rate (\%) } \\
\cline { 2 - 4 } \cline { 5 - 7 } Time, h & Control & siRNA-scr & siRNA-RBPJ & & Control & siRNA-scr & siRNA-RBPJ \\
\hline 0 & $1.55 \pm 0.12$ & 0 & 0 & & $2.58 \pm 0.21$ & 0 & 0 \\
24 & $1.60 \pm 0.06$ & $1.64 \pm 0.06$ & $1.48 \pm 0.14$ & & $3.32 \pm 0.21$ & $3.74 \pm 0.43$ & $9.55 \pm 1.65^{\mathrm{c}}$ \\
48 & $3.37 \pm 0.06$ & $3.45 \pm 0.18$ & $2.30 \pm 0.56^{\mathrm{a}}$ & & $4.04 \pm 0.61$ & $4.51 \pm 1.16$ & $16.93 \pm 1.41^{\mathrm{d}}$ \\
72 & $1.96 \pm 0.21$ & $1.26 \pm 0.39$ & $0.49 \pm 0.09^{\mathrm{b}}$ & & $4.27 \pm 0.39$ & $5.00 \pm 0.52$ & $25.71 \pm 3.95^{\mathrm{e}}$ \\
\hline
\end{tabular}

Data are presented as the mean \pm standard deviation. ${ }^{a} \mathrm{P}=0.0303$ vs. $48 \mathrm{~h}$ control; ${ }^{b} \mathrm{P}=0.0004$ vs. $72 \mathrm{~h}$ control; ${ }^{~} \mathrm{P}=0.0029$ vs. $24 \mathrm{~h}$ control; ${ }^{\mathrm{d}} \mathrm{P}=0.0001$ vs. $48 \mathrm{~h}$ control; ${ }^{\mathrm{e}} \mathrm{P}=0.0007 \mathrm{vs} .72 \mathrm{~h}$ control. siRNA, small interfering RNA; RPBJ, recombinant signal binding protein of immunoglobulin $\kappa \mathrm{J}$ region; scr, scrambled control.

was significantly correlated with the clinical index of PNH (Table IV; Fig. 1C and D). Upon screening and analyzing the sequencing results, the highly expressed and first-class mutation gene RBPJ was evaluated in depth. In total, $69.23 \%$ (9/13) of patients had RBPJ gene mutations, including non-synonymous, splicing and frame-shift mutations. The mutation sites were as follows: c.-57-2A $>\mathrm{C}$ (exon 2), c.163T $>$ A (exon 12), c.174C $>$ A (exon 12) and c.177T $>$ A (exon 12).

Expression level of RBPJ mRNA is increased in 30 patients with PNH and significantly correlates with clinical data. To obtain $\mathrm{CD} 9^{-} / \mathrm{CD} 9^{+}$neutrophils from $30 \mathrm{PNH}$ patients and $\mathrm{CD} 9^{+}$neutrophils from 30 healthy controls, immunomagnetic beads cell sorting was performed in peripheral blood of patients with PNH and healthy controls on the same day of blood extraction. The RT-qPCR results of CD59- and CD59+ neutrophils were as follows: The RBPJ expression levels in the CD59- cell group were markedly higher than that of the

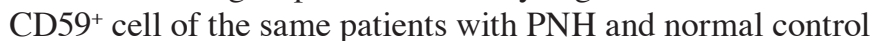
groups $(9.54 \pm 4.12$ vs. $2.37 \pm 0.65$ and $1.09 \pm 0.03$, respectively; $\mathrm{P} 1=0.0153$ and $\mathrm{P} 2=0.0074)$, and the difference between the CD59+ cell group and the normal group was not statistically significant $(\mathrm{P}=0.1305$; data not shown).

The correlation between the proportion of PNH clones and clinical hemolysis indicators was analyzed, and the results revealed that the $\mathrm{PNH}$ clone proportion was negatively correlated with the levels of $\mathrm{Hb}$ (Fig. 1D; $\mathrm{r}=-0.6359, \mathrm{P}=0.0002$ ), and positively correlated with Ret and LDH levels (Fig. 1D; $\mathrm{r}=0.5063, \mathrm{P}=0.0043$ and $\mathrm{r}=0.4066, \mathrm{P}=0.0258$, respectively). This indicated that the clone size of PNH is directly correlated with the clinical hemolysis index.

The correlation between the expression of RBPJ mRNA and hemolysis indicators was also analyzed. The results revealed that the expression of RBPJ mRNA was negatively correlated with $\mathrm{Hb}$ levels $(\mathrm{r}=-0.7334, \mathrm{P}=0.0074)$; positively correlated with the percentage of Ret $(\mathrm{r}=0.4223, \mathrm{P}=0.0201)$; positively correlated with LDH levels $(\mathrm{r}=0.4521, \mathrm{P}=0.0121)$; and positively correlated with $\mathrm{PNH}$ clones $(\mathrm{r}=0.4757$, $\mathrm{P}=0.0091$; Fig. 1E). These results indicate that the expression level of the RBPJ gene is associated with the hemolytic index in patients with $\mathrm{PNH}$.
Table VI. Comparison of paroxysmal nocturnal hemoglobinuria primary cell cycle distribution before and after transfection of siRNA RBPJ.

\begin{tabular}{lccc}
\hline Phase & Control (\%) & siRNA-scr $(\%)$ & siRNA-RBPJ (\%) \\
\hline G0/G1 & $66.95 \pm 6.42$ & $66.67 \pm 4.89$ & $81.66 \pm 4.04^{\mathrm{a}}$ \\
S & $16.45 \pm 2.08$ & $16.92 \pm 1.53$ & $6.707 \pm 1.53^{\mathrm{b}}$ \\
G2 & $16.60 \pm 8.18$ & $16.40 \pm 6.21$ & $11.64 \pm 2.52^{\mathrm{c}}$ \\
\hline
\end{tabular}

Data are presented as the mean \pm standard deviation. ${ }^{\mathrm{a}} \mathrm{P}=0.0285$ vs. $\mathrm{G} 0 / \mathrm{G} 1$ control; ${ }^{\mathrm{b}} \mathrm{P}=0.0028$ vs. $\mathrm{S}$ control; ${ }^{\mathrm{c}} \mathrm{P}=0.0285$ vs. $\mathrm{G} 2$ control. siRNA, small interfering RNA; RPBJ, recombinant signal binding protein of immunoglobulin $\mathrm{\kappa J}$ region; scr, scrambled control.

Proliferation of PNH clones is inhibited upon RBPJ gene knockdown by siRNA constructs in vitro. A total of 3 siRNA sequences (siRNA1, siRNA2 and siRNA3) were designed to transfect PNH primary cells in conjunction with lipo3000 to determine their potential capacity of silencing the expression of RBPJ (Fig. 2). The mRNA expression level of RBPJ in the transfected cells was detected by RT-qPCR. The 3 siRNAs were able to significantly inhibit the expression of RBPJ. Of them, RBPJ-siRNA1 was the most effective, as it reduced the expression of RBPJ in PNH primary cells by $>75 \%$ (Fig. 2A). Therefore, siRBPJ-1 was selected to inhibit the expression of RBPJ in PNH primary cells.

RBPJ-siRNA1 was transfected into PNH primary cells, which were then cultured in medium and harvested 24-72 h later. The levels of RBPJ were decreased in transfected PNH primary cells according to the results of RT-qPCR (Fig. 2B). These data suggest that, upon transfection of siRNA targeting the RBPJ1 gene into PNH primary cells, the RBPJ gene was silenced, and its mRNA expression level was reduced by $>75 \%$.

To clarify whether the growth of PNH primary cells was affected by RBPJ inhibition, CCK-8 assay was used to analyze the growth ability of PNH primary cells (Table V). Compared with the controls, RBPJ inhibition was detected $48 \mathrm{~h}$ following siRNA transfection, which significantly reduced cell growth (Fig. 2C). Cell apoptosis rate was analyzed at 24-72 h after transfection (Table V), and the rate of cell apoptosis increased 
following siRNA-RBPJ transfection, gradually increasing with transfection time (Fig. 2E and F). These results further suggested that inhibition of RBPJ may prevent the growth of PNH primary cells by inhibiting cell proliferation.

The cell cycle phases were analyzed $72 \mathrm{~h}$ following inoculation (Table VI). The results revealed that, compared with the control and scr-transfected cells, the percentage of S-phase proliferating cells in RBPJ knockdown PNH primary cells decreased markedly, while the percentage of G0/G1-phase cells increased (Fig. 2D). These results suggest that inhibition of RBPJ may prevent the growth of PNH primary cells by inhibiting cell proliferation.

\section{Discussion}

PNH is a benign clonal disease of hematopoietic stem cells, the pathogenesis of which remains unclear. PNH clones have immune-escape characteristics $(23,24)$ and anti-apoptotic properties $(25,26)$, and second gene mutations (27-32) may be involved in the amplification of PNH clones. Next-generation sequencing (NGS) achieves genetic heterogeneity due to the combination of somatic mutations and complex clonal structure, reflecting the sequence of genetic defects, and it is currently widely used in leukemia and other malignancies (33-36). This sequencing technology has the following advantages: i) The vast majority of disease variations in all exome regions of the human genome can be detected; and ii) it can detect common and low-frequency (i.e., mutation frequency $<5 \%$ ) mutations that cannot be detected by Sanger sequencing.

Previous sequencing results revealed that genetic mutations such as TET2, SUZ12, CUX1, RBPJ, MAGEC1 and MLL2 were identified as the key driving factor for the evolution of myeloid tumors or cancer clones $(10,11,37-40)$. The selection and evolution of genetic clones serves a role in malignant tumors and benign hematological diseases. The mutational events identified in the present study are not unique to $\mathrm{PNH}$, but significantly overlap with the spectrum of mutations observed in myeloid neoplasms and other solid tumors.

In vitro experiments on the highly expressed and first-class mutated RBPJ gene revealed that the expression of this gene in CD59- cells was significantly higher than that in CD59+ cells and controls. Furthermore, its expression was negatively correlated with $\mathrm{Hb}$ levels, and positively correlated with the percentage of Ret, LDH levels and PNH clones. These findings suggest that the abnormal expression and mutation of the RBPJ gene may be closely correlated with PNH clones. As the key transcription factor of the Notch signaling transduction pathway, RBPJ can mediate the transcriptional activation of the 4 Notch receptors in the nucleus and serve a pivotal role in the integration of Notch signaling pathways, thus being important in the regulation of the Notch signaling pathway $(41,42)$. The Notch-RBPJ signaling pathway serves a pivotal role in the differentiation of $\mathrm{T}$ cells in the thymus. The elimination of RBPJ in early thymus cells can decrease the differentiation $\alpha \beta$ T cells and increase the differentiation of $\gamma \delta \mathrm{T}$ cells $(43,44)$.

In the resent study, the expression of the RBPJ gene was validated in patients with PNH and controls, and this gene was confirmed to be highly expressed in PNH clones. This increased expression may be an epiphenomenon or even the consequence (rather than the cause) of PIG-A mutations. The expression of the RBPJ gene may be positively or negatively regulated by a GPI-linked protein, which may be upregulated or downregulated. Therefore, RBPJ gene silencing was conducted in PNH primary cells in vitro, and the changes in the apoptosis rate and proliferation index were evaluated to explore the role of the RBPJ gene in PNH.

Previous studies on the Notch-RBPJ signaling pathway in mice $(42,45)$ revealed that mutation of the Notch receptor and its downstream key transcription factor RBPJ may be teratogenic to mouse embryos or lead to early mortality due to failure of nerve tube closure. The results also revealed that the Notch/RBPJ signaling transduction pathway was involved in the growth and development of embryos. Fetal development is one of the key and pivotal factors regulating the differentiation and proliferation of neural progenitor cells. The expression of the RBPJ gene in lung and prostate cancer cells was previously silenced by Lv et al (46) and Xue et al (47), and the results suggested that the downregulation of RBPJ expression leads to a significant decrease in the growth of cancer cells, which suggests that RBPJ serves a downstream role in the Notch signaling pathway and appears to be essential for the functional activation of Notch signaling.

In the present study, siRNA technology was used to silence the RBPJ gene in PNH primary cells. Subsequently, the proportion of cells in the G0/G1 phase increased; the proportion of S-phase cells decreased; cells were arrested in G0/G1 stage; the apoptosis rate increased; and the cell proliferation ability decreased. This suggested that high expression of the RBPJ gene may be correlated with the proliferation of abnormal $\mathrm{PNH}$ clones.

In conclusion, besides mutations in PIG-A, the RBPJ gene is a highly expressed and first-order mutant gene screened by NGS, and its abnormal expression may contribute to the proliferation of $\mathrm{PNH}$ clones.

\section{Acknowledgements}

Not applicable.

\section{Funding}

The present study was supported by grants from National Natural Science Foundation of China (grant nos. 81770110, 81570106 and 81600093), the Tianjin Municipal Natural Science Foundation (grant nos. 18JCYBJC27200 and 15JCYBJC24300) and Tianjin Health and Family Planning Commission (grant no. 16ZXMJSY00180).

\section{Availability of data and materials}

All data generated or analyzed during our study are included in this article.

\section{Authors' contributions}

RF designed the research and revised the manuscript. LiyL performed the experiments, analyzed the data and wrote the manuscript. HL, HW, ZL, YC, LijL, JS and GW contributed to 
the experimental work and the collection of patient data. All authors read and approved the final manuscript.

\section{Ethics approval and consent to participate}

All procedures performed in the present study were in accordance with the 1964 Helsinki declaration and its later amendments or comparable ethical standards, and were approved by the Ethics Committee of Tianjin Medical University (Tianjin, China). Informed consent was obtained from all individual participants included in the study.

\section{Patient consent for publication}

Not applicable.

\section{Competing interests}

The authors declare that they have no competing interests.

\section{References}

1. Rotoli B and Luzzatto L: Paroxysmal nocturnal hemoglobinuria. Semin Hematol 26: 201-207, 1989.

2. Luzzatto L: Recent advances in the pathogenesis and treatment of paroxysmal nocturnal hemoglobinuria. F1000Res 5: 209, 2016.

3. Parker CJ: The pathophysiology of paroxysmal nocturnal hemoglobinuria. Exp Hematol 35: 523-533, 2007.

4. Hill A, DeZern AE, Kinoshita T and Brodsky RA: Paroxysmal nocturnal haemoglobinuria. Nat Rev Dis Primers 3: 17028 , 2017.

5. Schrezenmeier H, Muus P, Socié G, Szer J, Urbano-Ispizua A, Maciejewski JP, Brodsky RA, Bessler M, Kanakura Y, Rosse W, et al: Baseline characteristics and disease burden in patients in the international Paroxysmal Nocturnal Hemoglobinuria registry. Haematologica 99: 922-929, 2014.

6. Socié G, Mary JY, de Gramont A, Rio B, Leporrier M, Rose C, Heudier P, Rochant H, Cahn JY and Gluckman E: Paroxysmal nocturnal hemoglobinuria: Long-term follow-up and prognostic factors. Lancet 348: 573-577, 1996.

7. de Latour RP, Mary JY, Salanoubat C, Terriou L, Etienne G, Mohty M, Roth S, de Guibert S, Maury S, Cahn JY, et al: Paroxysmal nocturnal hemoglobinuria: Natural history of disease subcategories. Blood 112: 3099-3106, 2008.

8. Li LY, Liu ZY, Liu H, Liu CY, Shao ZH and Fu R: Deep Sequencing of whole genome exon inParoxysmal Nocturnal Hemoglobinuria. Am J Hematol 92: E51-E53, 2017.

9. Ramdzan ZM and Nepveu A: CUX1, a haploinsufficient tumour suppressor gene over-expressed in advanced cancers. Nat Rev Cancer 14: 673-682, 2014.

10. Kridel R, Sehn LH and Gascoyne RD: Pathogenesis of follicular lymphoma. J Clin Invest 122: 3424-3431, 2012.

11. Montagner S, Leoni C, Emming S, Della Chiara G, Balestrieri C, Barozzi I, Piccolo V, Togher S, Ko M, Rao A, et al: TET2 regulates mast cell differentiation and proliferation through catalytic and non-catalytic activities. Cell Rep 15: 1566-1579, 2016.

12. Sugimori C, Padron E, Caceres G, Shain K, Sokol L, Zhang L, Tiu R, O'Keefe CL, Afable M, Clemente M, et al: Paroxysmal nocturnal hemoglobinuria and concurrent JAK2(V617F) mutation. Blood Cancer J 2: e63, 2012.

13. Fraiman YS, Cuka N, Batista D, Vuica-Ross M and Moliterno AR: Development of paroxysmal nocturnal hemoglobinuria in CALR-positive myeloproliferative neoplasm. J Blood Med 30: 107-110, 2016

14. Chen Y, Tao S, Deng Y, Song L and Yu L: Chronic myeloid leukemia transformation in a patient with paroxysmal nocturna hemoglobinuria: A rare case report with literature review. Int J Clin Exp Med 15: 8226-8229, 2015.

15. Tominaga R, Katagiri T, Kataoka K, Kataoka K, Wee RK, Maeda A, Gomyo H, Mizuno I, Murayama T, Ogawa S and Nakao S: Paroxysmal nocturnal hemoglobinuria induced by the occurrence of BCR-ABL in a PIGA mutant hematopoietic progenitor cell. Leukemia 30: 1208-1210, 2016.
16. Orent W, Mchenry AR, Rao DA, White C, Klein HU, Bassil R, Srivastava G, Replogle JM, Raj T, Frangieh M, et al: Rheumatoid arthritis-associated RBPJ polymorphism alters memory CD4+ T cells. Hum Mol Genet 25: 404-417, 2016.

17. Nagao H, Setoguchi T, Kitamoto S, Ishidou Y, Nagano S, Yokouchi M, Abematsu M, Kawabata N, Maeda S, Yonezawa S and Komiya S: RBPJ is a novel target for rhabdomyosar coma therapy. PLoS One 7: e39268, 2012.

18. Parker C, Omine M, Richards S, Nishimura J, Bessler M, Ware R, Hillmen P, Luzzatto L, Young N, Kinoshita T, et al: Diagnosis and management of paroxysmal nocturnal hemoglobinuria. Blood 106: 3699-3709, 2006.

19. Livak KJ and Schmittgen TD: Analysis of relative gene expression data using real-time quantitative PCR and the 2(-Delta Delta C(T)) method. Methods 25: 402-408, 2001.

20. Kalejta RF, Shenk T and Beavis AJ: Use of a membrane-localized green fluorescent protein allows simultaneous identification of transfected cells and cell cycle analysis by flow cytometry. Cytometry 29: 286-291, 1997.

21. Shen W, Clemente MJ, Hosono N, Yoshida K, Przychodzen B, Yoshizato T, Shiraishi Y, Miyano S, Ogawa S, Maciejewski JP and Makishima $\mathrm{H}$ : Deep sequencing reveals stepwise mutation acquisition in paroxysmal nocturnal hemoglobinuria. J Clin Invest 124: 4529-4538, 2014.

22. Richards S, Aziz N, Bale S, Bick D, Das S, Gastier-Foster J, Grody WW, Hegde M, Lyon E, Spector E, et al: Standards and guidelines for the interpretation of sequence variants: A joint consensus recommendation of the American College of Medical Genetics and Genomics and the Association for Molecular Pathology. Genet Med 17: 405-424, 2015.

23. Karadimitris A and Luzzatto L: The cellular pathogenesis of paroxysmal noctural hemogolbinuria. Leukemia 15: 1148-1152, 2001.

24. Dacie JV: Paroxysmal nocturnal haemoglobinuria. Proc R Soc Med 56: 587-596, 1963.

25. Brodsky RA, Vala MS, Barber JP, Medof ME and Jones RJ: Resistance to apoposis caused by PIG-A mutations in paroxysmal noctural hemogolbinuria. Proc Natl Acad Sci USA 94: 8756-8760, 1997.

26. Horikawa $\mathrm{K}$, Nakakuma $\mathrm{H}$, Kawaguchi $\mathrm{T}$, Iwamoto $\mathrm{N}$, Nagakura S, Kagimoto T and Takatsuki K: Apoptosis resistance of blood cells from patients with paroxysmal nocturnal hemoglobinuria, aplastic anemia, and myelodysplastic syndrome. Blood 90: 2716-2722, 1997.

27. Hansen NE and Killmann SA: Paroxysmal nocturnal hemoglobinuria in myelofibrosis. Blood 36: 428-431, 1970.

28. Luzzatto L, Familusi JB, Williams CK, Junaid TA, Rotoli B and Alfinito F: The PNH abnormality in myeloproliferative disorders: Association of PNH and acute erythremic myelosis in two children. Haematologica 64: 13-30, 1979.

29. Nishimura JI,Inoue N, Azenishi Y, Hirota T, Akaogi T, Shibano M, Kawagoe K, Ueda E, Machii T and Takeda J: Analysis of PIG-A gene in a patient who developed reciprocal translocation of chromosome 12 and paroxysmal nocturnal hemoglobinuria during follow-up of aplastic anemia. Am J Hematol 51: 229-233, 1996.

30. Inoue N, Izui-Sarumaru $T$, Murakami $Y$, Endo $Y$, Nishimura J, Kurokawa K, Kuwayama M, Shime H, Machii T, Kanakura Y, et al: Molecular basis of clonal expansion of hematopoiesis in 2 patients with Paroxysmal nocturnal hemoglobinuria. Blood 108: 4232-4236, 2006.

31. Brodsky RA: Paroxysmal nocturnal hemoglobinuria: Stem cells and clonality. Hematology Am Soc Hematol Educ Program: 111-115, 2008.

32. Katagiri T, Tominaga R, Kataoka K, Maeda A, Gomyo H, Mizuno I, Murayama T, Ogawa S and Nakao S: A cure for paroxysmal nocturnal hemoglobinuria using molecular targeted therapy specific to a driver mutation. Blood 126: 1215, 2015.

33. Cancer Genome Atlas Research Network; Ley TJ, Miller C, Ding L, Raphael BJ, Mungall AJ, Robertson A, Hoadley K, Triche TJ Jr, Laird PW, et al: Genomic and epigenomic landscapes of adult de novo acute myeloid leukemia. N Engl J Med 368: 2059-2074, 2013.

34. Leproust E: Target-enrichment strategies for next generation sequencing. MLO Med Lab Obs 44: 26-27, 2012.

35. Ng SB, Buckingham KJ, Lee C, Bigham AW, Tabor HK, Dent KM, Huff CD, Shannon PT, Jabs EW, Nickerson DA, et al: Exome sequencing identifies the cause of a mendelian disorder. Nat Genet 42: 30-35, 2010.

36. Warr A, Robert C, Hume D, Archibald A, Deeb N and Watson M: Exome sequencing: Current and future perspectives. G3 (Bethesda) 5: 1543-1550, 2015. 
37. Liu C, Shi X, Wang L, Wu Y, Jin F, Bai C and Song Y: SUZ12 is involved in progression of non-small cell lung cancer by promoting cell proliferation and metastasis. Tumour Biol 35: 6073-6082, 2014.

38. Kulic I, Robertson G, Chang L, Baker JH, Lockwood WW, Mok W, Fuller M, Fournier M, Wong N, Chou V, et al: Loss of the Notch effector RBPJ promotes tumorigenesis. J Exp Med 212: 37-52, 2015.

39. Wong CC, Martincorena I, Rust AG, Rashid M, Alifrangis C, Alexandrov LB, Tiffen JC, Kober C; Chronic Myeloid Disorders Working Group of the International Cancer Genome Consortium, Green AR, et al: Inactivating CUX1 mutations promote tumorigenesis. Nat Genet 46: 33-38, 2014.

40. Wienand $\mathrm{K}$ and Shires K: The use of MAGE C1 and flow cytometry to determine the malignant cell type in multiple myeloma. PLoS One 10: e0120734, 2015.

41. Mugnaini E and Floris A: The unipolar brush cell: A neglected neuron of the mammalian cerebellar cortex. J Comp Neurol 339, 174-180, 1994.

42. Nunzi MG, Birnstiel S, Bhattacharyya BJ, Slater NT and Mugnaini E: Unipolar brush cells from a glutamatergic projection system within the mouse cerebellar cortex. J Comp Neurol 434: 329-341, 2001.
43. Sotelo $\mathrm{C}$ and Changeux JP: Bergmann fibers and granular cell migration in the cerebellum of homozygous weaver mutant mouse. Brain Res 77: 484-491, 1974.

44. Gerhardt DM, Pajcini KV, D'altri T, Tu L, Jain R, Xu L, Chen MJ, Rentschler S, Shestova O, Wertheim GB, et al: The Notch1 transcriptional activation domain is required for development and reveals a novel role for Notch1 signaling in fetal hematopoietic stem cells. Gene Dev 28: 576-593, 2014.

45. Maillard I, Weng AP, Carpenter AC, Rodriguez CG, Sai H, Xu L, Allman D, Aster JC and Pear WS: Mastermind critically regulates Notch-mediated lymphoid cell fate decisions. Blood 104: 1696-1702, 2004.

46. Lv Q, Shen R and Wang J: RBPJ inhibition impairs the growth of lung cancer. Tumour Biol 36: 3751-3756, 2015.

47. Xue L, Li H, Chen Q, Wang Z, Zhang P, Chen H, Wang Z and Chong T: Inhibition of recombining binding protein suppressor of hairless (RBPJ) Impairs the growth of prostate cancer. Cell Physiol Biochem 36: 1982-1990, 2015.

(c) (i)() This work is licensed under a Creative Commons CY NG NO Attribution-NonCommercial-NoDerivatives 4.0 International (CC BY-NC-ND 4.0) License. 